\title{
Dissolution rate and bioavailability enhancement of co-ground mixtures of paliperidone, with different hydrophilic carriers
}

\author{
*Anuja Pandey, Bhabagrahi Rath, Anil Kumar Dwivedi \\ Dr. Reddy's Lab, Hyderabad, Andhra Pradesh, India 500034
}

\begin{abstract}
Co-ground mixtures of poorly water soluble drug Paliperidone (PAL) with different hydrophilic carriers [Polyvinylpyrrolidine (Plasdone K-25 and Plasdone S-630), Hydroxypropyl methyl cellulose (HPMC), Hydroxypropylcellulose (HPC) and Sodium alginate were prepared to improve the dissolution rate of PAL. Co-grinding with PVP, especially with PVP- S630 (Vinyl pyrrolidone/ vinyl acetate co-polymer), was more effective in reduction of particle size than milling of drug alone. DSC studies indicated that crystalline nature of drug was reduced after co-grinding with PVP grades as compared to their corresponding physical mixtures. The hydrophilic carriers other than PVP did not reduce the crystalline nature of the drug significantly. X-ray diffraction (XRD) was carried out for selected batches to confirm DSC results. Significant enhancement in dissolution rate as well as extent was observed with co-ground mixtures of drug and PVP. Among all the prepared batches in this study, co-ground mixture of PAL and Plasdone S-630 in 1:1 ratio showed best results in terms of extent of dissolution as well as dissolution rate in water. This effect was not only due to particle size reduction, but also loss of crystalline nature of the drug during co-grinding. PVP was found to be a better carrier among the different hydrophilic carriers used in the study for improving the dissolution characteristics of PAL. The extent of the mean plasma exposures of PAL was 7-fold higher in animals treated with co-ground mixture of PAL, Plasdone S630 (1:1) compared to animals treated with Pure PAL.
\end{abstract}

Key Words: Crystalline, amorphous, S 630, physical mixture, particle size, solubility.

\section{INTRODUCTION}

With more than $50 \%$ of the drugs that have been taken for development having poor aqueous solubility; solubility enhancement techniques has been in the forefront of product development. Various techniques have been employed thus far for improving the solubility and consequently the bioavailability of poorly soluble drugs especially those belonging to BCS class II. Of the various techniques employed, co-grinding is one of the simplest, cost effective and environment friendly technique to improve the solubility of the drugs (Sugimoto et al., 1998; Suzuki et al., 2001; Friedrich et al., 2005). The increase in the solubility by this technique is achieved by the particle size reduction. Co-grinding of the poorly soluble drug with various

\footnotetext{
*Corresponding Author:

Anuja Pandey

Junior Manager

Dr. Reddy's Lab, Hyderabad

Andhra Pradesh, India 500034

E-mail: welcome.a2000@gmail.com

Contact No.: +919920785787
}

kinds of hydrophilic polymers helps in solubilization and enhances the bio availability because the drug gets transformed in to an amorphous form in a polymer net work. Co- grinding can be done either in a dry or in wet condition (Takahashi et al., 1985; Miyamae et al., 1994). However, a common disadvantage one usually encounters with wet grinding or milling is partial dissolution of the drug which more often results in uncontrolled re crystallization (Ahuja et al., 2007; Yamamoto et al., 1976). In the past, investigators have successfully used co-grinding under dry conditions for improving the solubility of poorly soluble drugs (Florence et al., 1976; Etman and Nada., 1999; Nada, 1997; Garg et al., 2009).

Co-grinding of hydrophobic drugs with a hydrophilic polymer causes creation of hydrophobic surfaces and the presence of hydrophilic polymers improves the wettability of the newly created hydrophobic surfaces, there by resulting in improvement of dissolution (Liversidge and Cundy, 1995; Kubo et al., 1996). Paliperidone (PAL), a major 
metabolite of risperidone (9-hydroxyrisperidone), has been approved by the FDA for the treatment of schizophrenia since 2006 and belongs to BCS Class II. Not many reports are available in literature where solubility enhancement of PAL has been attempted.

Therefore, the present work was proposed to study the effect of co-grinding of PAL with various hydrophilic carriers like Polyvinylpyrrolidone (PVP), Hydroxypropyl methylcellulose (HPMC), Hydroxypropyl cellulose (HPC), and Sodium alginate as well as with different grade of PVP on particle size, solid state properties and dissolution performance.

In the present study, particle size for physical mixture as well as co-ground mixture of PAL with different hydrophilic carriers has been investigated. Solid state characterization of the drug-polymer binary system was carried out using DSC and XRD techniques.

\section{MATERIALS AND METHODS}

\section{Materials}

Paliperidone was obtained from Dr. Reddy's Laboratories, Hyderabad, India. PVP (Plasdone K-25 and Plasdone S630) and Sodium Alginate (Keltone LV) were generously gifted by ISP, Wayne, New Jersey, USA. Hydroxypropyl methyl cellulose (Pharmacoat 606) and Hydroxypropyl cellulose (Klucel EF) was purchased from Shinetsu, Japan and Aqualon, Hereuld, USA, respectively. All other chemicals used were of analytical grade.

\section{Methods}

\section{Preparation of co-ground mixtures}

PAL (5g) and polymers (5 and $10 \mathrm{~g}$ ) in drug to polymer ratio of $1: 1$ and 1:2 ratio were co-ground at $200 \mathrm{rpm}$ for 120 minutes using a Planetary ball mill (Model- PM 100 Retsch, Germany). 120 minutes consist 4 cycles each of 30 minutes. After completion of one cycle powder was removed from wall of the vessel with spatula for proper grinding.

\section{Preparation of physical mixture}

Corresponding physical mixtures (PM) were prepared by triturating in a mortar with a pestle for 30 minutes.

\section{Particle size measurement}

The particle size of prepared mixtures was determined by laser diffractometer (Scirocco 2000(A), Malvern Instruments, UK). The relative frequency of the diameter of the particle was obtained by calculation based on volume distribution. The particle size at $10 \%\left(\mathrm{~d}_{10}\right), 50 \%\left(\mathrm{~d}_{50}\right)$ and $90 \%\left(\mathrm{~d}_{90}\right)$ of total fraction was obtained. The particle size at $90 \%$ of total fraction was used as particle size. The values were the average of ten measurements.

\section{Differential scanning calorimetry}

Thermal curves of each sample were recorded by simultaneous Differential scanning Calorimeter (TA Instruments Q 1000). Each sample (approximately $2.5 \mathrm{mg}$ ) were scanned in hermetic pan made of aluminum at heating rate of $10^{\circ} \mathrm{C} / \mathrm{min}$ over the range of $50-220^{\circ} \mathrm{C}$ with an empty aluminum pan used as reference. Samples were heated under nitrogen atmosphere (flow rate of $\mathrm{N}_{2}-50 \mathrm{ml} / \mathrm{min}$ ).

\section{$X$-Ray diffraction}

Powder X-ray diffraction patterns were traced employing X-ray diffractometer (Model No. 3000, Seifert, Germany) for the samples, using Ni-filtered $\mathrm{Cu}-\mathrm{K}$ radiation, a voltage of $40 \mathrm{kV}$, a current of 30 $\mathrm{mA}$ radiation scattered in the crystalline regions of the sample, which was measured with a vertical goniometer. Patterns were obtained by using a step width of $0.04^{\circ} \mathrm{C}$ with a detector resolution in $2 \theta$ (diffraction angle) between $10^{\circ}$ and $50^{\circ}$ at ambient temperature.

\section{In vitro dissolution studies}

In-vitro dissolution testing employed the USP Apparatus II (VK 7010 Varian, USA) at $50 \mathrm{rpm}$ with $900 \mathrm{ml}$ of degassed water (DosaprepX, DOSA $\mathrm{TECH})$ at $37 \pm 0.5^{\circ} \mathrm{C}$. Six capsules of each batch containing powder sample equivalent to $6 \mathrm{mg} \mathrm{PAL}$ were tested. Sink condition was maintained in degassed water, Sample of the dissolution media were removed via an automated sampling system at predetermined time interval $(0,5,10,15,30,45,60$ $\mathrm{min}$ ) and simultaneously analyzed spectrophotometrically at $\lambda \max$ of $237 \mathrm{~nm}$ (Carry $50 \mathrm{UV}$ Spectrophotometer attached with Dissolution Apparatus). In vitro release studies of unmilled PAL (UP), Milled PAL (MP), Physical mixtures (PM) and Co ground mixtures $(\mathrm{CM})$ were carried out, data of dissolution study is shown as Mean $\pm \mathrm{SD}(\mathrm{n}=6)$. Time taken to achieve 30\% ( $\mathrm{t}_{30} \%$ ) and $60 \%\left(\mathrm{t}_{60} \%\right)$ drug release in dissolution medium were used for comparing the dissolution of drug from the various physical \& co-ground mixtures with the pure drug (both milled \& unmilled). 


\section{Pharmacokinetic study in rats}

The study was conducted at Advinus Therapeutics Pvt. Ltd., Bangalore, India after getting the Ethical Committee approval (Registration No.: 219/2009/CPCSEA, dated: 25-05-2009). In total 12 (6 per group) female Sprague Dawley rats (6-7 weeks old) weighing between 180-230 $\mathrm{g}$ were used for the study. All rats had free access to tap water and pelleted diet (Sniff rats pellet food, Ssniff Spezialdiaten, Germany). The rats were housed in a cage and maintained on a $12 \mathrm{~h}$ light/dark at room temperature $\left(21^{\circ}\right.$ to $\left.24^{\circ} \mathrm{C}\right)$ and relative humidity of 50 to $70 \%$ and acclimatized to study area conditions for at least 5 days before dosing. General and environmental conditions were strictly monitored. The room underwent 10 fresh air change cycles per h. Rats were implanted with cannula in the jugular vein for blood sampling. The surgery was performed two days before dosing under anesthesia. The animals were fasted at least $10 \mathrm{~h}$ prior to dose administration and for $4 \mathrm{~h}$ post dose with free access to water. Individual oral doses of the test and reference samples were prepared $(0.8 \mathrm{mg} / \mathrm{kg}$ free base $)$ and accurately weighed drug material was carefully transferred into the dosing syringe containing aliquot of gelatin gel. Transfer the sample into the syringe barrel was accomplished either using a butter-paper funnel/with a spatula; the funnel was weighed before and after transferring drug to account for any loss by sticking to funnel. Separate funnels were used to prepare each dose. After transfer of the drug material into the syringe, an aliquot of gelatin was placed on top of the drug powder, thus effectively sandwiching it between 2 layers of gelatin. The sample was attached to an oral feeding needle and administered into the stomach. After dosing, syringe was rinsed with $1 \mathrm{~mL}$ of water and dosed again. Serial blood samples $(250 \mu \mathrm{L})$ were withdrawn from the cannulated jugular vein at: Pre dose, $0.25,0.5,1,1.5,2,4,8,12$ and 24 h post-dosing and collected in labeled tubes containing $20 \mu \mathrm{L}$ of EDTA dipotassium dehydrate solution $(200 \mathrm{mM})$ per $\mathrm{ml}$ of blood as anticoagulant. Blood samples were held on ice until centrifuged at $10000 \mathrm{rpm} ; 4^{\circ} \mathrm{C}$ for $10 \mathrm{~min}$. plasma was transferred to individual Eppendorf tubes and stored below $-70^{\circ} \mathrm{C}$ until bioanalysis.

\section{Bioanalysis}

The samples were analyzed by combined reversed phase liquid chromatography tandem mass spectrometry (LC-MS/MS Model no: API 4000, Applied Biosystems, Foster city, USA) by multiple reaction monitoring (MRM) and Positive ionization mode. The samples were prepared for analysis by liquidliquid extraction using tert-butyl methyl ether (TBME). Chromatography was performed on a 250mm X 4.6mm Kromasil $\mathrm{C}_{18}$ Column (Thermo) using isocratic elution with $85: 150.05 \mathrm{M}$ ammonium acetate and methanol. PAL pure drug was used as the internal standard. Under these conditions, no interference was observed for both samples and pure drug. The standard curve was linear from $1 \mathrm{ng} / \mathrm{ml}$ to $1000 \mathrm{mg} / \mathrm{ml}$.

\section{Pharmacokinetic data analysis}

The area under the drug concentration-time curve from zero to $24 \mathrm{~h}$ (AUC $0-24 \mathrm{~h}$ ) and mean residence time (MRT) were calculated using non compartmental analysis (WinNonlin 2.1, Pharsight Corp., Mountain View, CA). The maximal plasma concentration of drug $\left(\mathrm{C}_{\max }\right)$ and the time to reach maximum plasma concentration $\left(\mathrm{T}_{\max }\right)$ were directly obtained from plasma data by one-way ANOVA and Bonferroni's multiple pair comparison tests. The differences in $\mathrm{T}_{\max }$ among the groups were tested by Kruskal-wallis test and Dunn's multiple pair comparison tests.

\section{RESULTS AND DISCUSSION}

\section{Particle size measurement}

Milling of drug, Plasdone K25 and Plasdone S630 resulted in significant change in the particle size of the drug $\left(\mathrm{d}_{90}\right)$ as shown in table 1 , while milling was not effective in reducing the particle sizes of HPMC, HPC \& Sodium alginate owing probably to their fibrous nature. The co-ground mixtures of drug with respective polymers showed a decrease in $\mathrm{d}_{90}$ than the corresponding physical mixtures. However, significant reduction in particle size was observed only for drug Plasdone (K25 \& S630) coground mixtures. Further increasing the polymer content (Plasdone K25 \& S630) did not alter the PSD of both the physical \& co-ground mixtures. However, in case of fibrous carriers (HPMC, HPC and sodium alginate) an increase in the polymer content resulted in an increase in the particle size (Table 1). 
Table 1: The particle size ( $d_{10}, d_{50}$ and $\left.d_{90}\right)$ of drug and polymers before and after grinding, physical mixtures and co-ground mixtures in 1:1 and 1:2 ratios of all polymers.

\begin{tabular}{|c|c|c|c|}
\hline Batch Name & $d_{10}(\mu \mathrm{m})$ & $d_{50}(\mu \mathrm{m})$ & $\mathrm{d}_{90}(\mu \mathrm{m})$ \\
\hline Unmilled PAL & $9.31 \pm 0.52$ & $55.54 \pm 1.89$ & $202.23 \pm 11.26$ \\
\hline Milled PAL & $4.57 \pm 0.02$ & $25.14 \pm 0.15$ & $108.53 \pm 2.29$ \\
\hline Unmilled Plasdone K-25 & $13.62 \pm 0.31$ & $47.10 \pm 1.05$ & $117.62 \pm 8.32$ \\
\hline Milled Plasdone K-25 & $3.52 \pm 0.1$ & $20.31 \pm 0.21$ & $47.63 \pm 0.35$ \\
\hline PM PAL : K-25 1:1 & $12.84 \pm 0.06$ & $49.87 \pm 0.22$ & $140.01 \pm 0.72$ \\
\hline CM PAL: K-25 1:1 & $1.64 \pm 0.01$ & $12.31 \pm 0.15$ & $54.44 \pm 2.17$ \\
\hline PM PAL: K-25 1:2 & $14.73 \pm 0.06$ & $50.64 \pm 0.171$ & $129.83 \pm 0.65$ \\
\hline CM PAL: K-25 1:2 & $1.22 \pm 0.06$ & $9.76 \pm 0.12$ & $46.23 \pm 0.64$ \\
\hline Unmilled Plasdone S-630 & $13.24 \pm 0.23$ & $42.21 \pm 0.34$ & $114.12 \pm 1.83$ \\
\hline Milled Plasdone S-630 & $4.32 \pm 0.05$ & $15.21 \pm 0.09$ & $47.63 \pm 0.72$ \\
\hline PM PAL: S-630 1:1 & $12.85 \pm 0.11$ & $46.21 \pm 0.72$ & $147.1 \pm 7.25$ \\
\hline CM PAL : S-630 1:1 & $1.47 \pm 0.05$ & $11.92 \pm 0.1$ & $51.38 \pm 1.26$ \\
\hline PM PAL: S-630 1:2 & $13.82 \pm 0.08$ & $46.24 \pm 0.27$ & $143.18 \pm 8.52$ \\
\hline CM PAL S-630 1:2 & $2.02 \pm 0.05$ & $10.01 \pm 0.03$ & $39.43 \pm 1.15$ \\
\hline Unmilled HPMC & $21.23 \pm 0.44$ & $70.12 \pm 0.09$ & $159.83 \pm 0.29$ \\
\hline Milled HPMC & $20.82 \pm 0.18$ & $66.42 \pm 0.16$ & $155.59 \pm 0.24$ \\
\hline PM PAL: HPMC 1:1 & $13.54 \pm 0.13$ & $59.43 \pm 0.82$ & $163.12 \pm 1.48$ \\
\hline CM PAL: HPMC 1:1 & $3.22 \pm 0.26$ & $44.12 \pm 1.04$ & $150.35 \pm 6.42$ \\
\hline PM PAL: HPMC 1:2 & $16.81 \pm 0.2$ & $64.36 \pm 0.18$ & $159.46 \pm 0.1$ \\
\hline CM PAL HPMC 1:2 & $5.45 \pm 0.12$ & $57.23 \pm 0.71$ & $192.66 \pm 10.42$ \\
\hline Unmilled HPC & $116.21 \pm 7.42$ & $351.30 \pm 9.63$ & $753.41 \pm 21.42$ \\
\hline Milled HPC & $125.66 \pm 4.10$ & $359.15 \pm 5.37$ & $744.36 \pm 14.43$ \\
\hline PM PAL: HPC 1:1 & $25.33 \pm 0.20$ & $218.35 \pm 3.32$ & $604.60 \pm 3.30$ \\
\hline CM PAL: HPC 1:1 & $11.87 \pm 0.34$ & $275.02 \pm 4.20$ & $718.3 \pm 31.19$ \\
\hline PM PAL: HPC 1:2 & $38.03 \pm 2.63$ & $270.47 \pm 12.21$ & $673.02 \pm 31.96$ \\
\hline CM PAL : HPC 1:2 & $40.21 \pm 1.21$ & $316.03 \pm 5.79$ & $768.83 \pm 48.36$ \\
\hline Unmilled Sod. Alginate & $19.09 \pm 0.12$ & $69.92 \pm 0.66$ & $163.46 \pm 5.3$ \\
\hline Milled Sod. Alginate & $22.04 \pm 0.457$ & $64.549 \pm 1.12$ & $169.39 \pm 8.32$ \\
\hline PM PAL: Sod. Alginate 1:1 & $12.90 \pm 0.10$ & $59.68 \pm 1.04$ & $183.96 \pm 10.01$ \\
\hline CM PAL: Sod. Alginate 1:1 & $3.24 \pm 0.1$ & $34.64 \pm 0.53$ & $146.12 \pm 10.53$ \\
\hline PM PAL: Sod. Alginate 1:2 & $15.10 \pm 0.1$ & $63.5 \pm 0.24$ & $168.24 \pm 0.59$ \\
\hline CM PAL: Sod. Alginate 1:2 & $3.80 \pm 0.10$ & $50.78 \pm 1.07$ & $186.10 \pm 9.42$ \\
\hline
\end{tabular}

When the drug alone is ball-milled, mechanically micronized substances are electrostatically charged and, in most cases, they are agglomerated due to their cohesive behavior (Villiers et al., 1996. The agglomeration of particles during ball-milling can be prevented by using homopolymer of PVP (Plasdone K25) and co-polymer of PVP (Plasdone S630) and cellulose ethers (HPMC, HPC). These polymers are adsorbed onto the newly created particle surfaces (Liversidge, 1997). In the present study, among all the polymers co-polymer of PVP (Plasdone S630) was more effective in stabilizing the system and result in effective particle size reduction.

\section{Differential scanning calorimetry (DSC)}

DSC studies were performed on the individual components and on freshly prepared co- ground and physical mixtures in order to study the interaction between PAL and the carriers in the solid state. PAL exhibited a single sharp melting endothermic peak at $181^{\circ} \mathrm{C}$. The DSC thermograms of hydrophilic poly-
Table 2: Dissolution parameters of PAL and prepared physical and co-ground mixtures.

\begin{tabular}{|c|c|c|}
\hline Batch & $\mathbf{t}_{30 \%}$ (minute) & $\mathbf{t}_{60 \%}$ (minute) \\
\hline Un milled PAL & NA & NA \\
\hline Milled PAL & 59.25 & NA \\
\hline PM PAL : K-25 1:1 & NA & NA \\
\hline CM PAL : K-25 1:1 & 26.43 & NA \\
\hline PM PAL : K-25 1:2 & NA & NA \\
\hline CM PAL : K-25 1:2 & 24.36 & NA \\
\hline PM PAL : S-6301:1 & NA & NA \\
\hline CM PAL : S-6301:1 & 19.54 & 48.26 \\
\hline PM PAL : S-6301:2 & NA & NA \\
\hline CM PAL : S-6301:2 & 26.21 & 53.21 \\
\hline CM PAL : HPMC 1:1 & NA & NA \\
\hline CM PAL : HPMC 1:2 & NA & NA \\
\hline CM PAL : HPC $1: 1$ & 50.24 & NA \\
\hline CM PAL : HPC 1:2 & NA & NA \\
\hline PM PAL:Sod.Alginate1:1 & NA & NA \\
\hline CM PAL : Sod.Alginate1:1 & NA & NA \\
\hline PM PAL : Sod.Alginate1:2 & NA & NA \\
\hline CM PAL : Sod.Alginate1:2 & NA & NA \\
\hline
\end{tabular}

mers (Plasdone K25, Plasdone S630, HPMC, HPC and Sodium Alginate (Keltone) showed a broad endothermic peak in range between $50-130^{\circ} \mathrm{C}$, which may be due to endothermic relaxation. The DSC thermograms indicate that polymers are amorphous and hydrated compound (Figure 1a to 1e).

In general, both the physical mixtures and coground mixtures showed evidence of reduced crystallinity of the drug. The extent of reduction in crystallinity was more pronounced in case of coground mixtures. Out of the polymers studied, Plasdones showed significant reduction in crystallinity of the drug in comparison to other polymers, not only in co-ground mixtures, but also in their corresponding physical mixtures. Out of the two grades of Plasdone studied, Plasdone S630 was found to be more effective in reducing the crystallinity of the drug (Figure 1a to 1e).

\section{X-Ray diffraction (XRD)}

X-Ray diffraction studies were undertaken to consolidate DSC data indicating reduction of crystallinity of PAL with PVP. Therefore, X-ray diffraction pattern of PAL (unmilled and milled), Plasdone S630, physical mixture and co-ground mixture in 1:1 ratio were observed. 


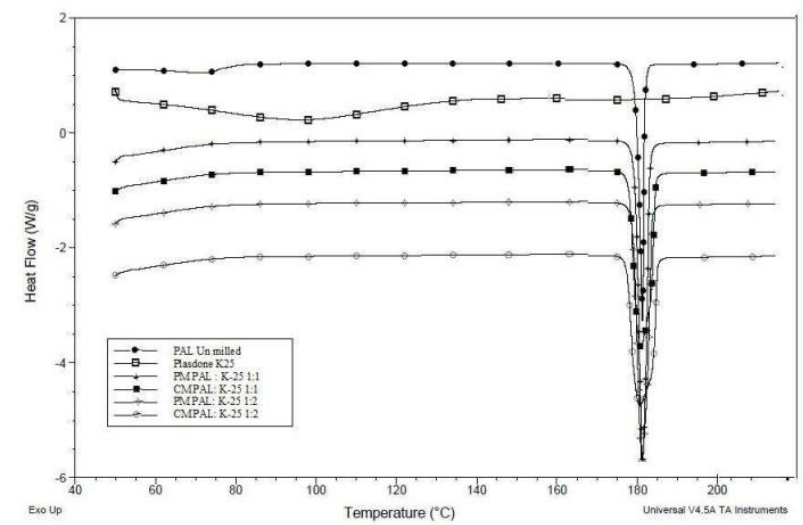

(a)

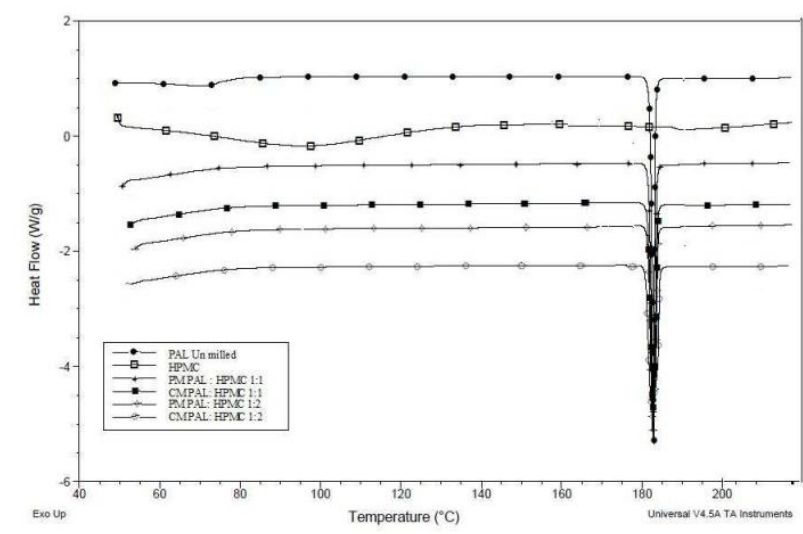

(c)

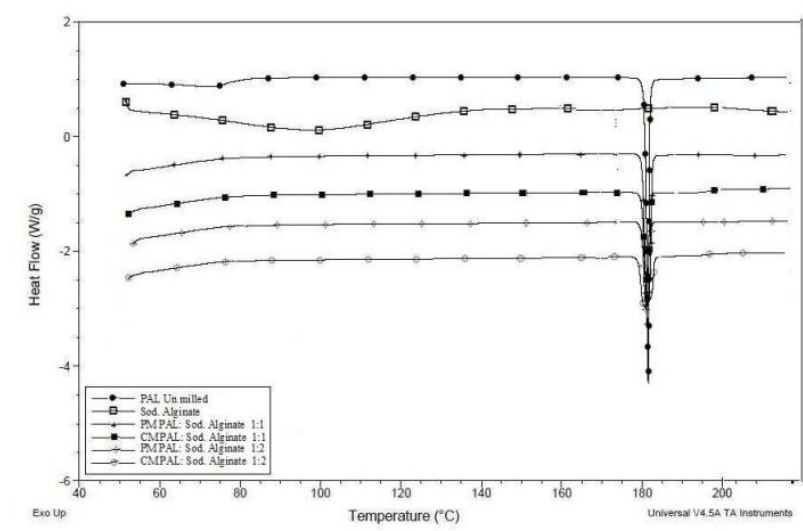

(e)

The diffraction spectrum of un-milled \& milled PAL was super impossible suggesting milling did not result in amorphization (Figure 2a, 2b). All the principal peaks of PAL were present in their physical mixtures and co-ground mixtures, although with lower intensity. No new peaks could be observed, suggesting the absence of interaction between the drug and the carrier. The prominent peaks of PAL at $2 \theta$ of $8.3,10.4,14.7,15.1,16.3,18.8,20.2,20.8,22.2$, $24.8,25.2$ and 28.1 were clearly seen at the same position in the physical mixture. However, in coground mixture, similar diffraction peaks were

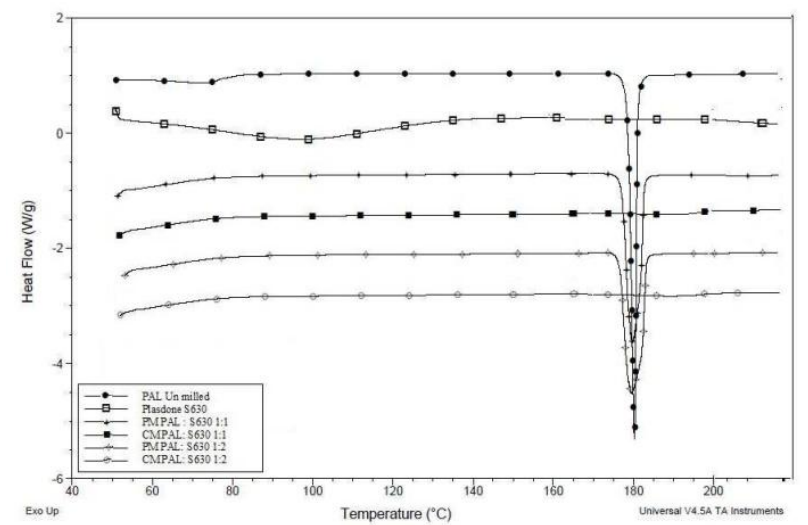

(b)

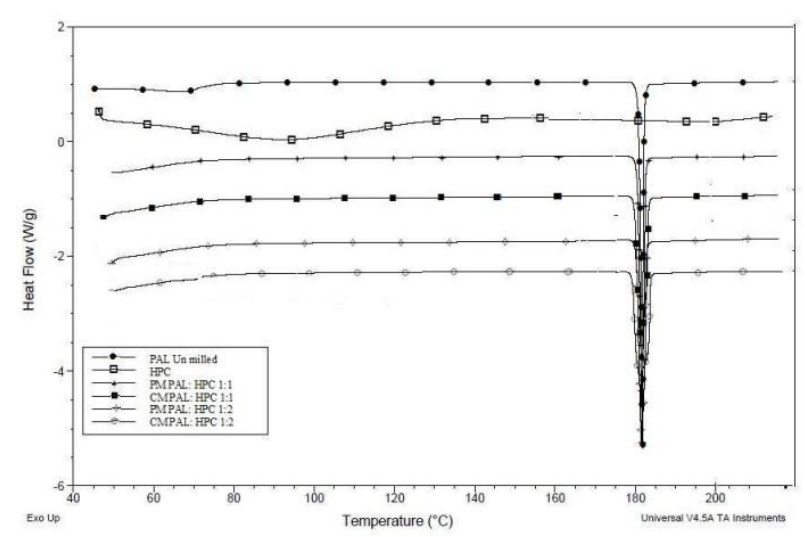

(d)

Figure 1: DSC Thermogram of -
(a) PAL and Plasdone K-25 Mixtures,
(b) PAL and Plasdone $S 630$ Mixtures,
(c) PAL and HPMC Mixtures,
(d) PAL and HPC Mixtures,
(e) PAL and Sodium Alginate Mixtures.

obtained at $2 \theta$ of $8.3,10.4,14.7,15.1,16.3,18.8,20.2$, 20.8, 22.2, 24.8, 25.2 and 28.1. These peaks were broadened and reduced in intensity in comparison to diffraction peaks of corresponding physical mixture (Figure 2c, 2d, 2e), suggesting that either the crystal quality is reduced or change is induced in the crystal orientation or only some of drug is still present in the crystalline form (Vippangunta et al., 2002; Valizadeh et al., 2004; Betageri et al., 1995). PAL therefore existed in a very less crystalline state in co-ground mixture with Plasdone S630. Corresponding Physical mixture showed higher degree of crystallinity than the co- 


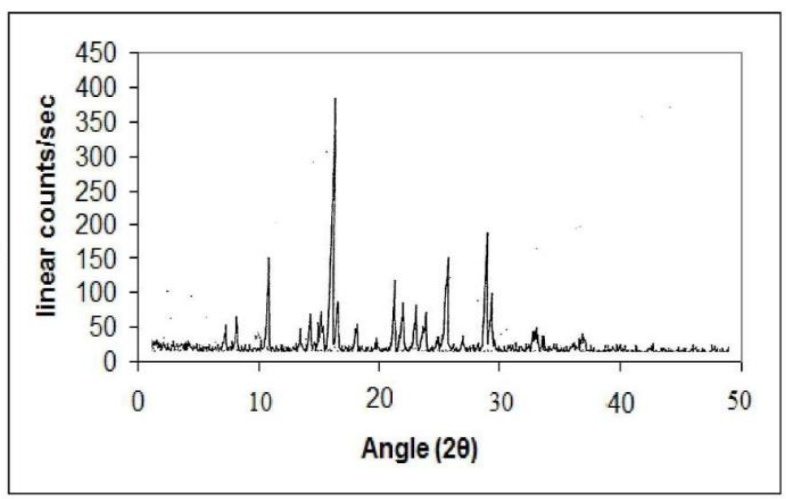

(a)

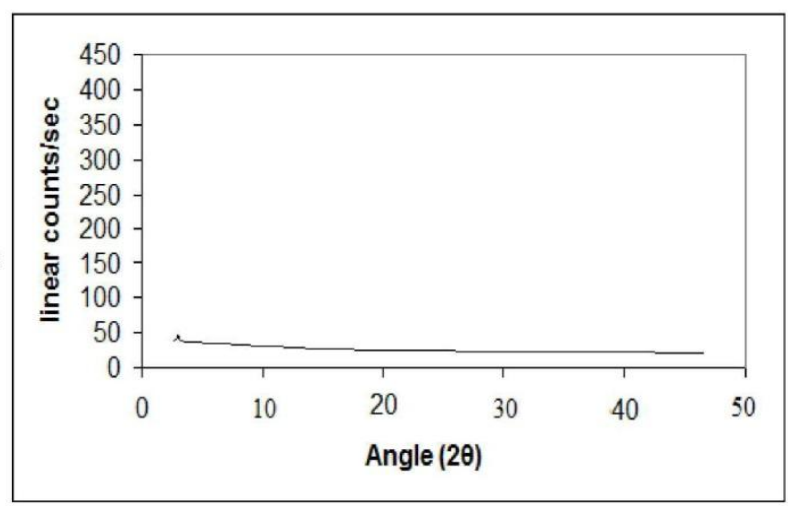

(c)

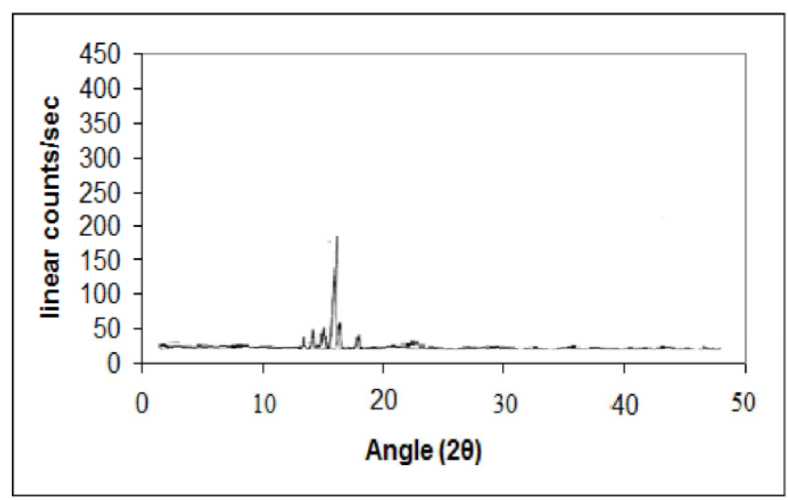

(e)

ground mixture, thus confirming the results of the DSC study.

\section{In-vitro dissolution studies}

Table 2 enlists the various dissolution parameters such as $\mathrm{t}_{30} \%$ and $\mathrm{t}_{60} \%$ of PAL, physical mixtures and co-ground mixtures of PAL with hydrophilic carriers used in the study. As it is evident from the table 2, unmilled drug showed less than 20\% dissolution in $1 \mathrm{~h}$ while milling of API resulted in marginal increase in dissolution. Overall the dissolution did not improve from either physical

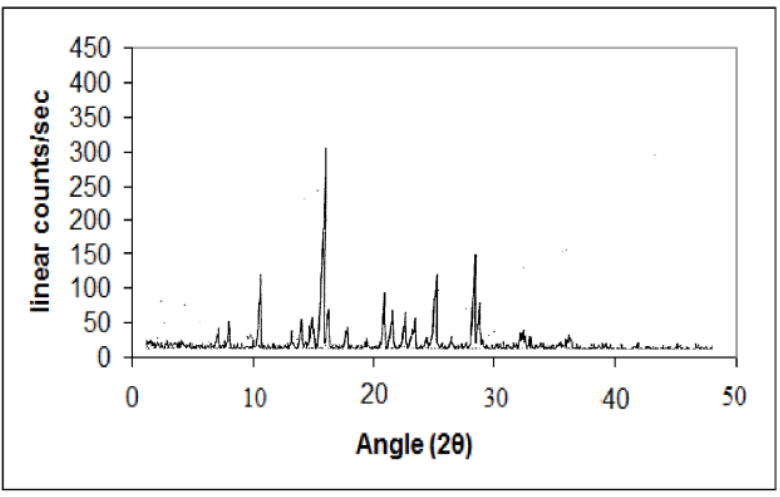

(b)

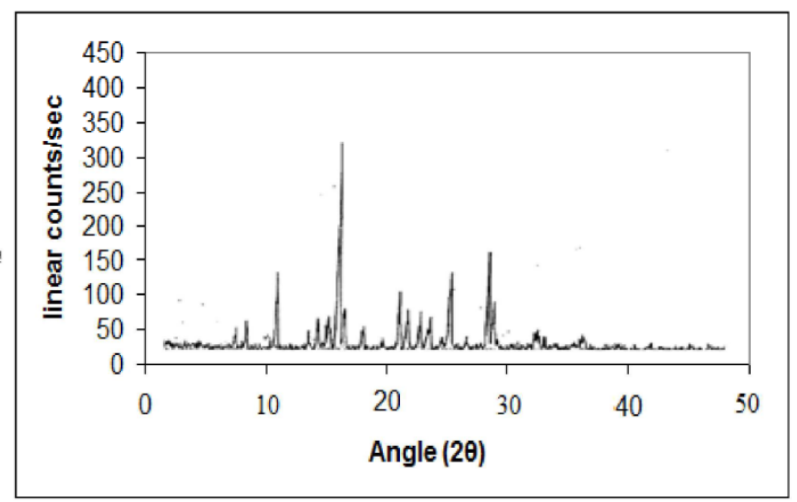

(d)

Figure 2: XRD Spectra of -
(a) Unmilled PAL
(b) Milled PAL
(c) Plasdone S-630
(d) PM PAL: S-630 1:1
(e) CM PAL: S-630 1:1

mixture or co-ground mixtures of drugs with HPMC, HPC and sodium alginate. Out of the two grades of PVP used plasdone S630 significantly enhanced the dissolution of PAL when compared to pure drug (milled \& unmilled; Figure 3a). Plasdone K25 mixtures though failed to achieve $60 \%$ dissolution within $1 \mathrm{~h}$, also showed significant increase in rate of dissolution when compared to pure drug (Figure 3b). Amorphous forms of pharmaceuticals are markedly more soluble than their crystalline counterparts (Hancock and Parks, 2000) and improve dissolution rate (Friedrich et al., 2005; 
Table 3: Pharmacokinetic parameters of PAL with $\mathbf{9 0 \%}$ confidence intervals (CI) in female Sprague Dawley rats following oral administration of co ground mixture and PAL Pure drug in $1 \%$ gelatin gel sandwich (Dose: $0.8 \mathrm{mg} / \mathrm{kg}$ free base).

\begin{tabular}{lllllll}
\hline Sample & Tmax (h) & $\begin{array}{l}\text { Cmax } \\
\text { (ng/mL) }\end{array}$ & $\begin{array}{l}\text { AUC0-last } \\
\text { (ng.h/mL) }\end{array}$ & $\begin{array}{l}\text { AUC0-inf } \\
\text { (ng.h/mL) }\end{array}$ & MRT0- t & T1/2 (h) \\
\hline PAL: Plasdone S 630 (1:1) & $2.4(1.4-4.3)$ & $242 \pm 162$ & $1189 \pm 412$ & $1289 \pm 407$ & $5.6 \pm 1.2$ & $0.8 \pm 1.9$ \\
Pure PAL & $8.2(4.2-8.5)$ & $14.9 \pm 4.60$ & $124 \pm 22.4$ & $176 \pm 13.5$ & $9.8 \pm 2.1$ & $8.8 \pm 2.8$ \\
\hline
\end{tabular}

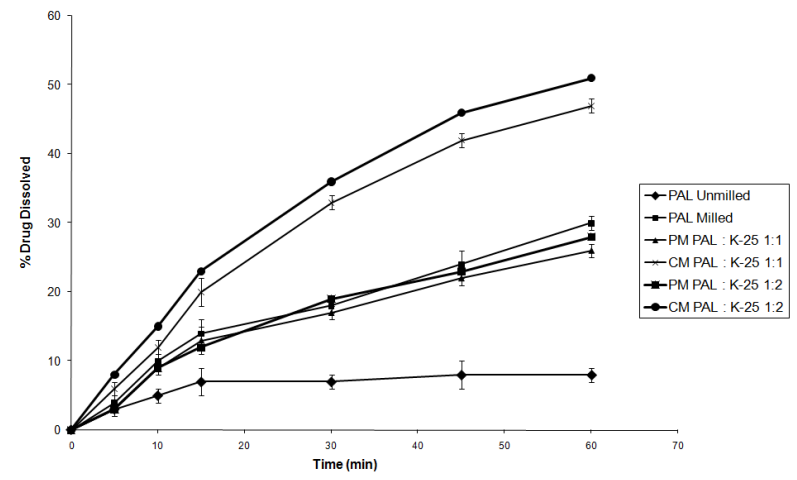

(a)

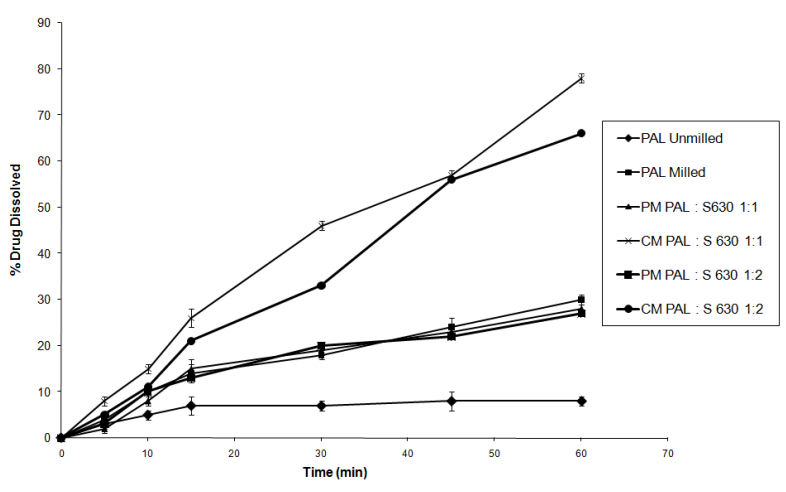

(b)

Figure 3: Dissolution Profiles of UP, MP, Physical mixture and co-ground mixtures of -

(a) Plasdone K25 in water (b) Plasdone S630 in water.

Ahuja et al., 2007). In case of co-ground mixtures with plasdone S630, it was surprising to note a decrease in release rate of PAL from 1:2 co-ground mixtures. This could probably be explained due to leaching out of the polymer from co-ground mixtures containing higher proportion of polymer during dissolution, which could form a concentrated layer of the polymer around the drug particles; therefore, the migration of drug particles into the bulk of dissolution medium would be slowed (Akbuga et al., 1988; Ford et al., 1986).

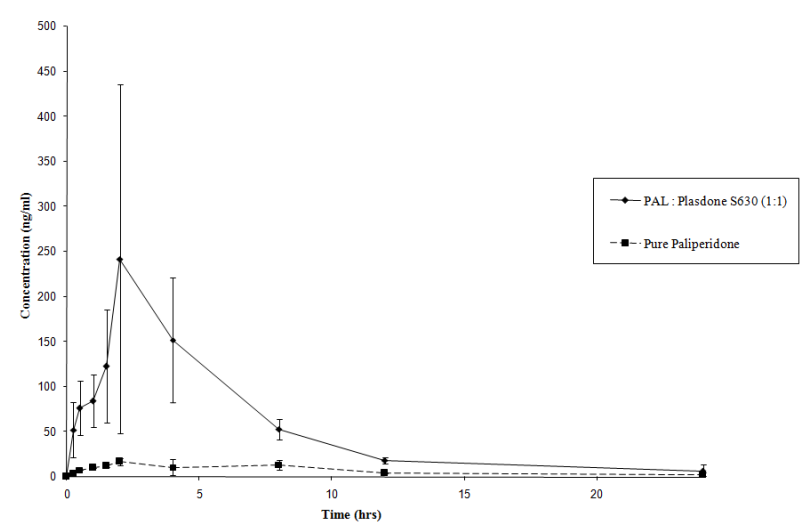

Figure 4: Plasma concentration-time data of co-milled PAL : Plasdone S630 (1:1) and pure PAL samples in female Sprague Dawley rats following oral administration in $1 \%$ gelatin gel sandwich (Dose: $0.8 \mathrm{mg} / \mathrm{kg}$ free base).

\section{Pharmacokinetic study}

The pharmacokinetic parameters of PAL were determined after oral administration of PAL and coground mixture of PAL with Plasdone S630 in the ratio of 1:1. The co-ground PAL: S-630 in ratio of 1:1 was selected on the basis of in-vitro dissolution studies as discussed above. The Plasma concentration time data of PAL are shown in figure 4 and their mean pharmacokinetic parameters are shown in table 3.

The extent of the mean Plasma exposures of PAL was 7 fold higher in animals treated with co-ground mixture of PAL compared to animals treated with PAL pure drug. Thus, the mean Plasma AUC 0 -last in animals that received co-ground mixture of PAL and PAL was $1189 \pm 412 \mathrm{ng} \cdot \mathrm{h} / \mathrm{mL}$ and $124 \pm 22.4$ ng.h/mL respectively, and they were significantly different ( $p=0.0001$ by ANOVA). Bonferroni's multiple pair comparison tests showed significant increase with co-ground as compared to PAL.

The corresponding mean Cmax values for these treatment groups were $242 \pm 162 \mathrm{ng} / \mathrm{ml}$, and $14.9 \pm 4.6$ $\mathrm{ng} / \mathrm{ml}$ and these were significantly different $(\mathrm{p}=$ 0.0052 by ANOVA). Bonferroni's multiple pair comparison tests showed significant increase with co-ground mixture compared to PAL. The median Tmax of PAL in animals that received the co-ground 
mixture and pure drug was 2.4 and $8.2 \mathrm{~h}$, respectively, and these were significantly different $(\mathrm{p}=0.0211$ by Kruskal-Wallis test). Dunn's multiple pair comparison tests also showed significant difference between the groups administered with the coground mixture to the group administered with the pure drug. The mean elimination half-life calculated from the pure drug administered animals was not reliable as there was insufficient number of time points on the terminal declining phase.

\section{CONCLUSION}

Cogrinding of PAL with Plasdone S630, reduced drug crystallinity, increased the rate and extent of dissolution, and improved bioavailability. Co-grinding of PAL, especially with Plasdone S630 showed immense promise in terms of not only increasing the dissolution but also in exposure in rat model. However, further studies are required before adopting co-grinding with Plasdone S630 as an alternate strategy for improving the bioavailability of PAL.

\section{REFERENCES}

Ahuja, N., Katare, O.P., Singh, B. (2007) Studies on dissolution enhancement and mathematical modeling of drug release of poorly water-soluble drug using water soluble carriers. Eur. J. Pharm. Biopharm. 65: 26-38. [DOI] PMid:16962750

Akbuga, J., Gursoy, A., Kendi, E. (1988). The preparation and stability of fast release furosemide- PVP solid dispersion. Drug Dev. Ind. Pharm. 14: 1439-1464. [DOI]

Betageri, G.V., Makarla, K R. (1995). Enhancement of dissolution of glyburide by solid dispersion and lyophilization techniques, Int. J. Pharm. 126: 155-160. [DOI]

Etman, M.A., Nada, A.H. (1999). Evaluation of simple and ballmill ground mixtures of two NSAIDs with hydrophilic carriers, Alexandria J. Pharm. Sci. 13: 135-140.

Florence, A.T., Salole, E.G. (1976). Changes in crystallinity and solubility on comminution of digoxin and observations on pironolactone and oestradiol, J. Pharm. Pharmacol. 28(8): 637642. [DOI] PMid:11314

Ford, J.L. (1986). The current status of solid dispersions, Pharm. Acta Helv. 61: 69-88.

Friedrich, H., Nada, A., Bodmeier, R. (2005) Solid state and dissolution rate characterization of co- ground mixtures of nifedipine and hydrophilic carriers. Drug Dev. Ind. Pharm. 31: 719-728. [DOI] PMid:16221606

Garg, A., Singh, S., Rao, V.U., Bindu, K., Balasubramaniam, J. (2009). Solid state interaction of raloxifene $\mathrm{HCl}$ with different hydrophilic carriers during co-grinding and its effect on dissolution rate. Drug Dev. Ind. Pharm. 35(4):455-70. [DOI] PMid:19048425

Hancock, B.C., Parks, M. (2000). What is true solubility advantage for amorphous pharmaceuticals? Pharm. Res. 17(4): 397-403. [DOI] PMid:10870982

Kubo, H., Osawa, T., Takashima, K., Mizobe, M. (1996). Enhancement of oral bioavailability and pharmacological effect of 1-(3,4-dimethoxyphenyl)-2, 3-bis (methoxycarbonyl)4- hydroxy-6,7,8-trimethoxynaphthalene (TA-7552), a new hypocholesterolemic agent, by micronization in co-ground mixture with D-mannitol. Biol Pharm Bull. 19(5):741-7. [DOI] PMid:8741587

Liversidge, G.G. (1997). Formulation of Oral Gastrointestinal Diagnostic X-ray Contrast Agents and Oral Gastrointestinal Therapeutic Agents, U. S. Patent US 5628981.

Liversidge, G.G., Cundy, K.C. (1995). Particle size reduction for improvement of oral bioavailability of hydrophobic drugs: I. Absolute oral bioavailability of nanocrystalline danazol in beagle dogs. Int. J. Pharm. 125: 91-97. [DOI]

Miyamae, A., Kema, H., Kawabata, T., Yasuda, T., Otsuka, M., Matsuda, Y. (1994). X-ray powder diffraction study on the grinding effect of the polymorphs of a novel and orally effect uricosuric agent: FR760505, Drug Dev. Ind. Pharm. 20: 28812887. [DOI]

Nada, A.H. (1997). Evaluation of ball-mill ground mixtures of hydrophilic carriers and phenytoin. Alexandria J. Pharm. Sci. 11: 29-33.

Sugimoto, M., Okagaki, T., Narisawa, S., Koide, Y., Nakajima, K. (1998). Improvement of dissolution characteristics and bioavailability of poorly water-soluble drugs by novel cogrinding method using water-soluble polymer. Int. J. Pharm. 160: 11-19. [DOI]

Suzuki, H., Ogawa, M., Hironaka, K., Sunada, H. (2000). A nifedipine co-ground mixture with sodium deoxycholate. I. Colloidal particle formation and solid state analysis. Drug Dev. Ind. Pharm. 27(9): 943-9. [DOI] PMid:11763472

Takahashi, Y., Nakashima, T., Ishihara, K., Nakagawa, H., Sugimoto, I. (1985). Drug Dev. Ind. Pharm. 11: 1543-1549. [DOI]

Valizadeh, H., Nokhodchi, A., Qarakhani, N., Zakeri-Milani, P., Azarmi, S., Hassanzadeh, D., Lobenberg, R. (2004). Physicochemical characterization of solid dispersions of indomethacin with PEG 6000, myrj 52, lactose, sorbitol, dextrin, and eudragit $^{\circledR}$ E100. Drug Dev. Ind. Pharm. 30: 303-317. [DOI] PMid:15109030

Villiers, De.M.M., Tiedt, L.R. (1996). An analysis of fine grinding and aggregation of poorly soluble drug powders in a vibrating ball mill. Pharmazie. 51: 564-567.

Vippangunta, S.R., Maul K.A., Tallavajhala, S., Grant, D.J.W. (2002). Grant. Solid state characterization of nifedipine solid dispersion. Int. J. Pharm. 236: 111-126. [DOI]

Yamamoto, K., Nakano, M., Arita, T., Takayama, Y,. Nakai, Y. (1976). Dissolution behavior and bioavailability of phenytoin from a ground mixture with microcrystalline cellulose, J. Pharm. Sci. 65(10): 1484-1488. [DOI] PMid:978407 